\title{
União de Juntas Dissimilares Alumínio-Aço de Chapas Finas pelo Processo de Soldagem por Atrito com Pino não Consumível (SAPNC)
}

\author{
(Dissimilar Joint of Aluminum-Steel Thin Sheet by Friction Stir Welding Process)
}

\author{
Edwar Andrés Torres ${ }^{1-2}$, Antonio Jose Ramirez ${ }^{1}$ \\ ${ }^{1}$ Laboratório Nacional de Luz Síncrotron - LNLS, Campinas, São Paulo, Brasil, elopez@lnls.br \\ ${ }^{2}$ Universidade Estadual de Campinas - UNICAMP -DEMA, Campinas, São Paulo, Brasil
}

\begin{abstract}
Resumo
Foram obtidas juntas dissimilares da liga de alumínio 6063-T5 e do aço AISI SAE 1020 com espessura de 2,0 mm soldadas por atrito com pino não consumivel. O objetivo deste trabalho é avaliar o efeito da penetração e o deslocamento da ferramenta na obtenção de juntas soldadas Al-aço. As juntas foram avaliadas segundo a qualidade da superfície e a profundidade da região soldada. Foi determinado que além das velocidades de rotação ( $\omega$ ) e avanço (v), o deslocamento e a profundidade de penetração da ferramenta são parâmetros fundamentais, pois definem o aporte térmico e, com este, a aderência ou não de alumínio na ferramenta, a qualidade superficial e a formação de defeitos ao longo da linha da junta.
\end{abstract}

Palavras-chave: Soldagem por atrito com pino, juntas dissimilares Al-aço, parâmetros de soldagem.

Summary: Aluminum alloy 6063-T5 and AISI SAE 1020 were obtained with dissimilar welded joints by friction stir welding in 2.0 $\mathrm{mm}$ thick sheet. The aim was to evaluate the effect of welding parameters to obtain Al-steel welds. Joints were evaluated according to surface quality and depth of the welded region. It was determined that besides the rotational ( $\omega$ ) and forward (v) speeds, the offset and the tool penetration are key parameters because they define the heat input and therefore the adherence of aluminum in the tool, the surface quality and defect formation along the joint line.

Keywords: Friction Stir Welding, dissimilar joints Al-steel, welding parameters.

\section{Introdução}

A redução de peso nos veículos é uma preocupação constante na indústria automotiva, visando diminuir o consumo de combustível mantendo os padrões de segurança. Nesta procura, a soldagem de materiais mais leves, como alumínio, representa um desafio importante, já que ainda muitos dos sistemas envolvidos nos veículos são construídos a partir de materiais como o aço. É por isto que o desenvolvimento de uniões dissimilares alumínioaço se tornou imprescindível para a indústria do transporte. Os processos tradicionais de soldagem por fusão derivam na formação de compostos intermetálicos (CIM) do tipo $\mathrm{Fe}_{x} \mathrm{Al}$, diminuindo significativamente a tenacidade da junta [1]. Outros processos como a soldagem a laser, difusão, ultrassom e laminação já foram empregados com resultados promissores, entretanto persiste a formação dos compostos já citados [2]. A soldagem por atrito com pino não consumível (SAPNC) Friction Stir Welding (FSW) - vem sendo utilizada na união de sistemas complexos devido à ausência de fusão e excelente reprodutibilidade do processo, sendo aplicada a soldagem de sistemas dissimilares, incluindo a soldagem de alumínio e aço $[3,4]$.

(Recebido em 28/03/2011; Texto final em 01/09/2011).
$\mathrm{Na}$ SAPNC de juntas dissimilares alumínio-aço, alguns trabalhos simbólicos como Jiang e Watanabe [2,4], reportam o sucesso da SAPNC na junção de ambos os materiais, sendo recorrentes em citar a formação na interface entre os dois metais de uma camada fina de CIM do tipo $\mathrm{Fe}_{x} A l_{y}$. Fukumoto [5] determinou como a resistência da junta soldada aumenta a medida que a ferramenta entra mais no aço. Uzun [6] observou a formação da zona termo-mecanicamente afetada (ZTMA) em ambos os lados da junta ao soldar a liga 6013-T4 e o aço inoxidável X5CrNi18-10. Outro sistema semelhante - liga de alumínio 6053 e aço inox 304 - foi estudado por Lee [7] que determinou a formação de uma banda de grãos ultrafinos e alongados de ferrita e uma camada de compostos intermetálicos $\mathrm{Al}_{4} \mathrm{Fe}$ de $250 \mathrm{~nm}$ de espessura. Chen realizou a seleção da velocidade de avanço e rotação tomando como referência o efeito destes na energia absorvida por impacto Charpy na junta soldada $[8,9]$. Os mesmos parâmetros foram testados por Yasui [10] para determinar sue efeito no fluxo de material e a formação de defeitos, desta vez usando velocidade de avanço acima de $1000 \mathrm{~mm} \cdot \mathrm{s}^{-1}$, usando ferramenta de carbeto de tungstênio (WCCo). O estudo permitiu determinar parâmetros a partir dos quais era inibida a formação de CIM. Esses resultados contrastam com os obtidos por Tanaka [11] que determinou a existência de CIM para as mesmas condições que o Yasui. Nessa pesquisa o Tanaka relaciona o aumento na espessura do CIM com a entrada de calor, e a conseqüente redução nas propriedades mecânicas. 
Resultados similares foram obtidos por Soundararajan [12] ao soldar chapas de 6,0 mm de Al 6061-T6 e aço AISI 1018, empregando ferramenta de tungstênio rênio (W-25Re). O fluxo de material durante a soldagem de juntas a topo e sobrepostas foi estudado por Coelho $[13,14]$ usando EBSD de alta resolução, comprovando a assimetria do fluxo, a formação de vórtices no lado de avanço, a não mistura dos materiais e a existência de um processo de recuperação e recristalização heterogêneo na interface.

Apesar da literatura declarar o sucesso na obtenção das juntas soldadas Al-aço, estas se centram na determinação de parâmetros e suas respostas nas propriedades mecânicas e microestruturais. A literatura descarta o aspecto superficial da junta soldada, e o mais crítico, a obtenção de uma junta homogênea livre de defeitos na raiz, como elemento fundamental para avaliar os parâmetros de soldagem. O próximo é um trabalho exploratório para a determinação de parâmetros para a soldagem de juntas dissimilares Al-aço de 2,0 $\mathrm{mm}$ de espessura pelo processo SAPNC, usando como critérios de avaliação o aspecto superficial e a obtenção de junta com penetração total.

\section{Materiais e Métodos}

Os materiais utilizados foram chapas de $500 \times 85 \times 2,0$ $\mathrm{mm}$ da liga de alumínio 6063-T5 e de aço AISI SAE 1020. A composição química para a liga 6063 foi medida através de espectroscopia de emissão por plasma (Inductively Coupled
Plasma-optical Emission Spectroscopy - ICP-OES). Na Tabela 1 são apresentadas as composições químicas para a liga 6063 segundo a norma UNS No. A96063 e o resultado da composição medida através de ICP-OES.

A soldagem foi realizada usando uma máquina exclusiva para SAPNC, a qual é mostrada na Figura 1a, e uma ferramenta com matriz metálica e reforço cerâmico (Figura 1b) de carbeto de tungstênio (WC-12Co) com um ombro e pino de 25 e 5,7 $\mathrm{mm}$ de diâmetro, respectivamente, e comprimento de pino de $1,35 \mathrm{~mm}$.

Os parâmetros de soldagem empregados foram $300 \mathrm{rpm}$ de velocidades de rotação $(\omega)$ e $150 \mathrm{~mm} \cdot \mathrm{min}^{-1}$ de velocidade de avanço (v). A diferença entre a soldagem de juntas do mesmo metal e juntas dissimilares é que nesta última foi estabelecido um novo parâmetro conhecido como deslocamento da ferramenta (offset), o qual corresponde à distância da tangente do pino à linha da junta, sendo representado esquematicamente na Figura 2 [7]. Todos os parâmetros e variáveis são descritas na Tabela 2. Na soldagem de juntas dissimilares, o lado de avanço corresponde ao material de maior dureza, neste caso, o aço 1020, como mostrado na Figura 2c. A soldagem foi realizada em um único passe de $450 \mathrm{~mm}$ de comprimento, sendo modificado o deslocamento da ferramenta a cada $150 \mathrm{~mm}$. As juntas soldadas foram submetidas a dobramento para determinar a profundidade atingida na região da soldagem.

Tabela 1. Composição química liga de alumínio 6

\begin{tabular}{|c|c|c|c|c|c|c|c|c|}
\hline & $\mathbf{S i}$ & $\mathbf{F e}$ & $\mathbf{C u}$ & $\mathbf{M n}$ & $\mathbf{M g}$ & $\mathbf{C r}$ & $\mathbf{Z n}$ & $\mathbf{T i}$ \\
\hline Norma & $0,20-0,6$ & 0,35 & 0,10 & 0,10 & $0,45-0,9$ & 0,10 & 0,10 & 0,10 \\
\hline Medida & 0,46 & 0,13 & 0,02 & 0,03 & 0,39 & - & - & 0,01 \\
\hline
\end{tabular}
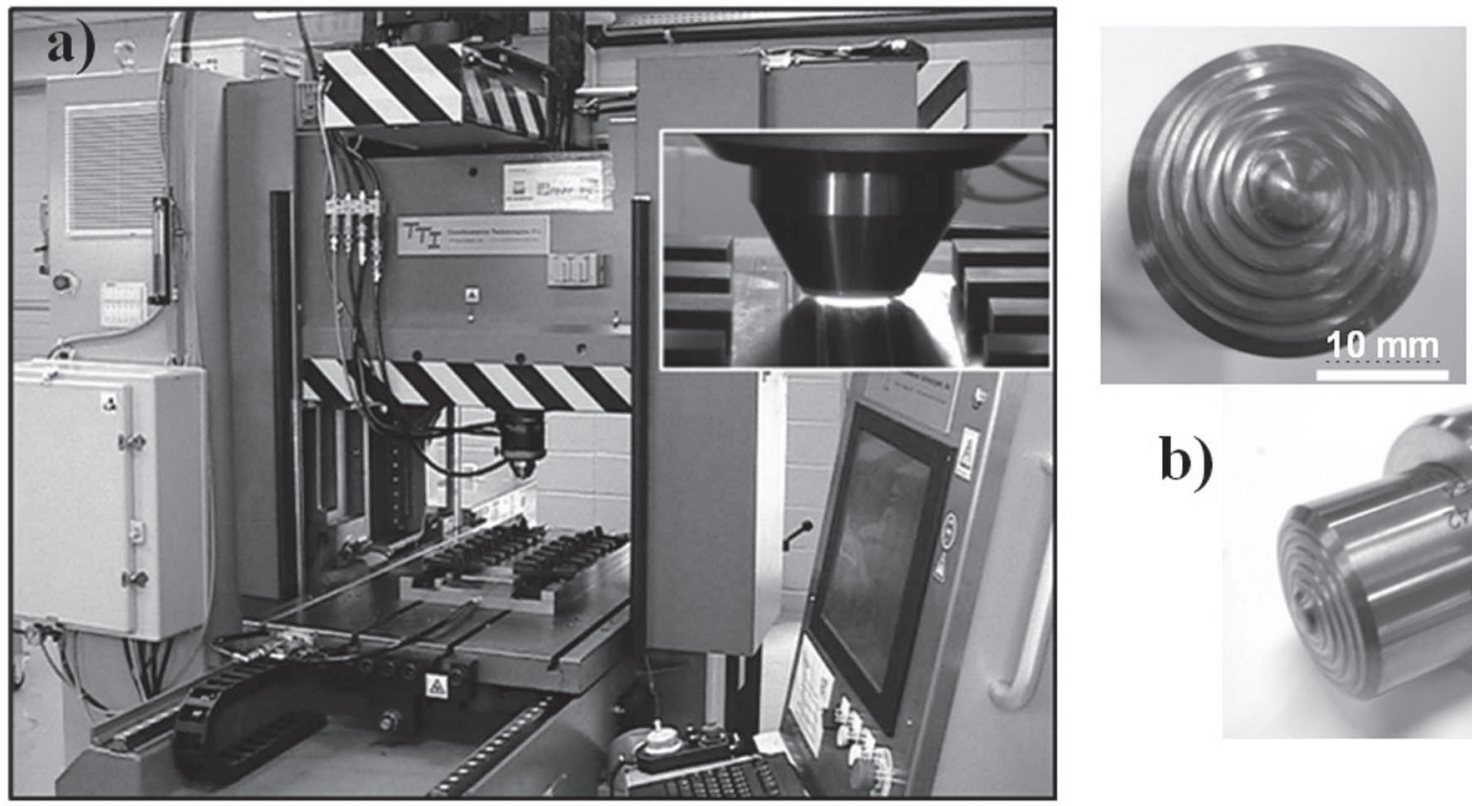

\section{WC-12Co}

Figura 1. a) Equipamento para soldagem FSW, b) Ferramentas de PCBN e WC utilizadas no projeto. 
Tabela 2. Parâmetros de soldagem AA 6063 / Aço 1020.

\begin{tabular}{|c|c|c|c|c|}
\hline Junta & $\begin{array}{c}\omega \\
{[\mathbf{r p m}]}\end{array}$ & $\begin{array}{c}v \\
{[\mathrm{~mm} / \mathrm{min}]}\end{array}$ & $\begin{array}{c}\text { Deslocamento } \\
{[\mathrm{mm}]}\end{array}$ & $\begin{array}{c}\text { Penetração } \\
\text { [mm] }\end{array}$ \\
\hline (1) & \multirow{3}{*}{300} & \multirow{3}{*}{150} & \multirow{2}{*}{0,00} & 1,50 \\
\hline (2) & & & & \multirow{2}{*}{1,70} \\
\hline (3) & & & 0,20 & \\
\hline
\end{tabular}

As juntas soldadas foram avaliadas segundo o aspecto da superfície e a profundidade consolidada na junta soldada. A penetração total da solda foi evidenciada por meio de um teste não convencional de dobramento e de uma medida de penetração. Esta medida e a caracterização microestructural foram realizadas por meio de microscopia ótica (MO); a caracterização microestrutural foi complementada com imagens obtidas por microscopia eletrônica de varredura (MEV). As amostras foram preparadas usando lixa com granulometria de 100 a 1500, seguido de polido com diamante e sílica de 1,0 $\mu \mathrm{m}$. Para observar a microestrutura inicialmente as amostras foram atacadas com nital $2 \%$, seguido de ataque com ácido fluorídrico (HF) $1 \%$.

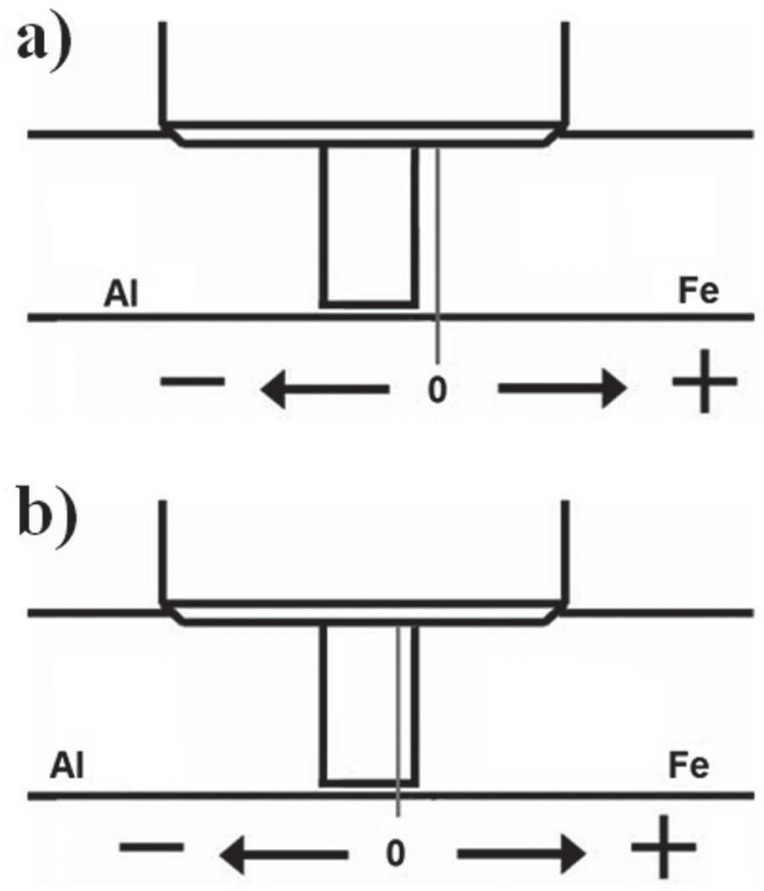

considerados responsáveis pelas propriedades mecânicas da família $6 \mathrm{XXX}$, correspondem a compostos submicrométricos $\mathrm{Mg}_{2} \mathrm{Si}$ [171819-20]. No entanto, estes precipitados foram identificados mediante X-EDS como do tipo $\alpha_{\mathrm{AlFeSi}}$ (Figura 3d). Os parâmetros de soldagem estudados são o deslocamento e a penetração da ferramenta, os quais foram avaliados considerando três critérios: o aspecto da superfície soldada, a penetração na junta e o estado da ferramenta.

As juntas soldadas são apresentadas na Figura 4a, destacando o estado da superfície soldada, as imagens dos testes de dobramento, e as macrografias da seção transversal das juntas. A Figura 4a-(1) corresponde à superfície da junta soldada com os parâmetros (1) da Tabela 2. Apesar de o acabamento ser adequado, o teste de dobramento mostra a abertura severa da raiz, evidenciando falta de penetração, o que é comprovado na imagem da macro, na região demarcada pelo quadro vermelho. Como era de se esperar, a medida que aumenta a penetração da ferramenta a região soldada se torna maior, como mostrado na junta da Figura 4a-(2), mas ainda sem penetração total. No entanto, é clara a perda de qualidade superficial, a qual coincide com a aderência de alumínio na ferramenta, formando uma crosta espessa como mostrado na Figura 5a. Finalmente, a condição
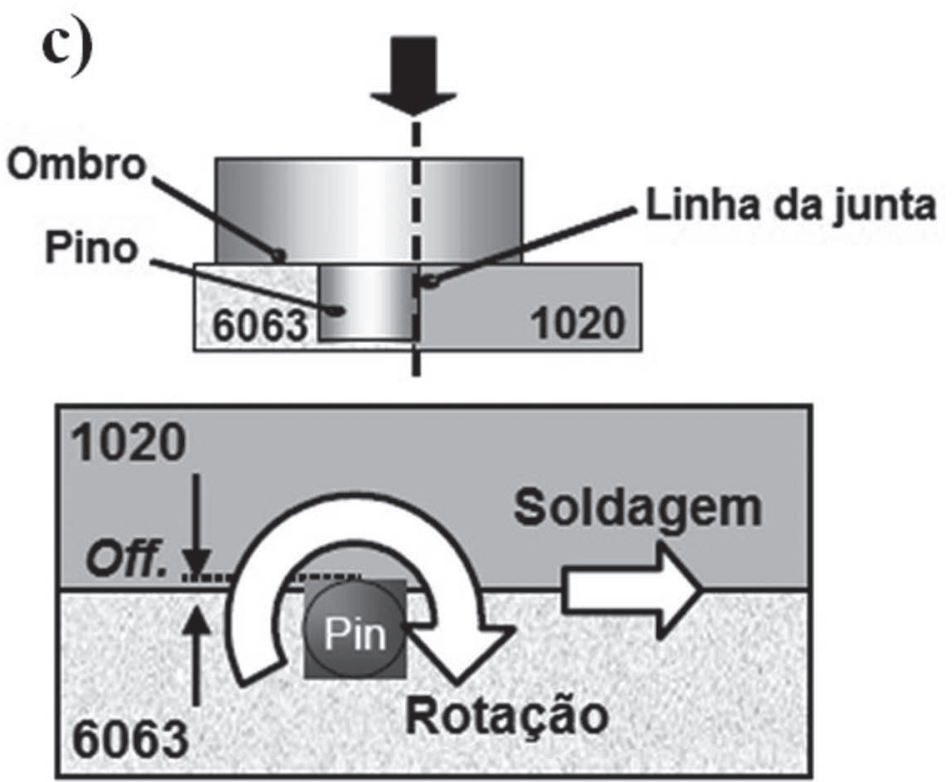

Figura 2. a) Deslocamento negativo com o pino embutido completamente no alumínio, b) deslocamento positivo com o pino entrando tangencialmente no aço, c) deslocamento zero e esquema da montagem da junta dissimilar. A linha vermelha representa a interface entre os dois materiais.

\section{Resultados e Discussão}

A caracterização microestrutural da liga 6063 revela a matriz $\alpha_{\mathrm{Al}}$ decorada por partículas - mais claras - como pode ser visto na Figura 3a-b. Estas partículas de comprimento entre 0,2 a 5,0 $\mu \mathrm{m}$ e aparentemente cilíndricas, são na realidade placas como evidencia a Figura 3c. Existe pouca informação acerca destas partículas $[15,16]$ já que os precipitados mais importantes, de soldagem representada na Figura 4a-(3) mostra a obtenção de uma junta com penetração total, mas com uma superfície soldada precária. Esta junta foi obtida com a mesma penetração da condição (2), mas com um deslocamento da ferramenta maior. Portanto, existe uma relação entre o deslocamento da ferramenta e a penetração na junta soldada, o qual é fundamental no momento de considerar a geometria da ferramenta para a soldagem de juntas dissimilares. 

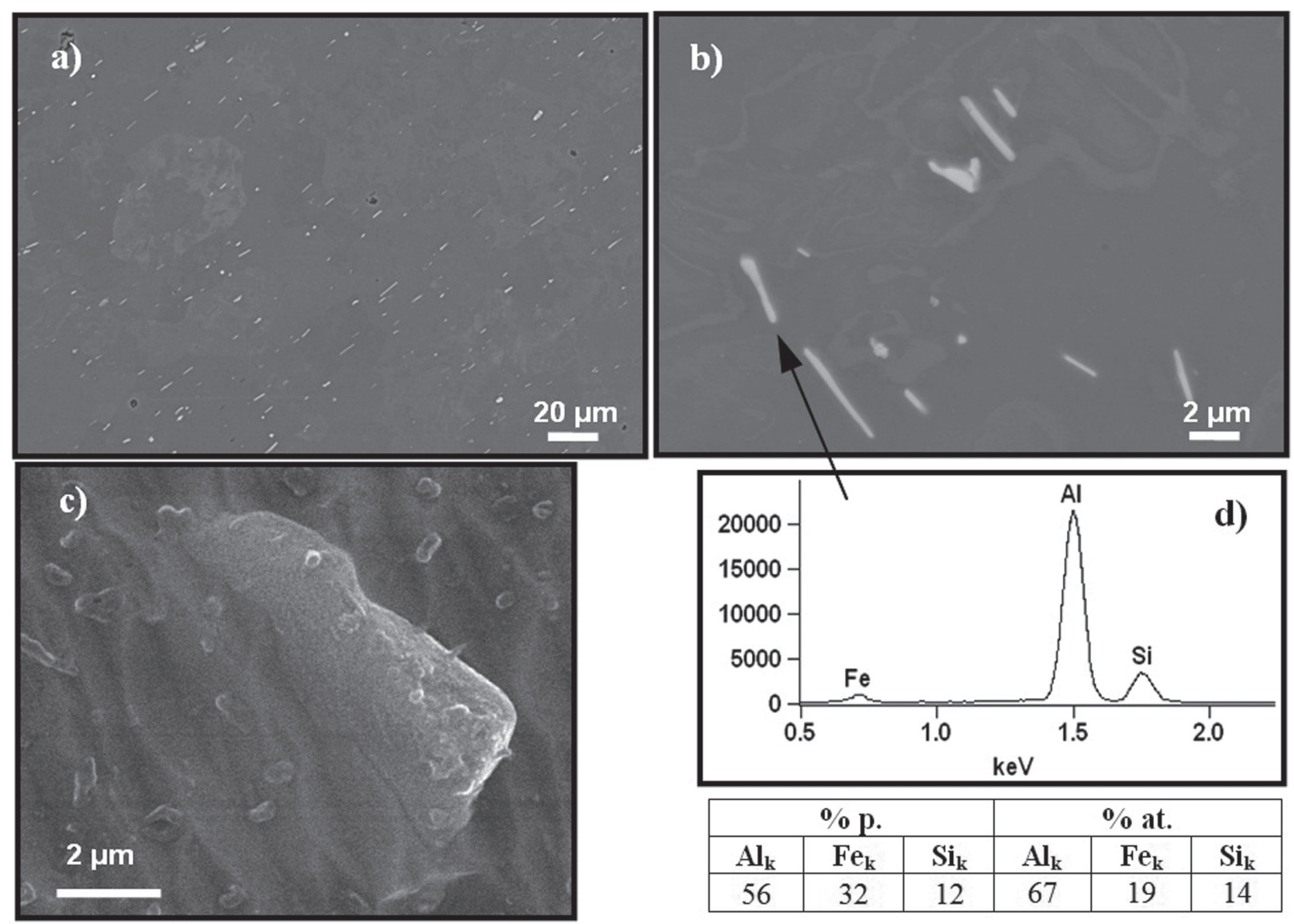

Figura 3. Caracterização microestrutural da liga 6063-T5 via MEV. a) Matriz com precipitados, b) detalhe dos precipitados aparentemente cilíndricos, c) imagem dos precipitados evidenciando o formato real de chapas, d) espectro de raios X (X-EDS) identificando os precipitados como do tipo $\alpha_{\mathrm{AlFeSi}}$.

Indícios do acontecido à ferramenta durante a aderência do alumínio na soldagem são observados na Figura 5 b; duas regiões na crosta de alumínio aderido à ferramenta são formadas. A superfície brilhante é atribuída à maior entrada de calor durante o processo de soldagem, por possível microfusão [21]. Um parâmetro que governa a quantidade de calor gerado é a área de contado entre o ombro da ferramenta e a junta. Esta área, em parte, está definida pela geometria da ferramenta e pela penetração, para os quais foi elaborado o esquema da Figura 4b, penetração de 1,5 e $1,7 \mathrm{~mm}$.

O aumento na área de contato, por efeito da penetração, gera mais calor, plastificando ainda mais o alumínio que termina sendo depositado na ferramenta. Isso acarreta não só em uma superfície de acabamento ruim, com vazios ao longo da linha da junta, como também na perda significativa de espessura na região da junta soldada. Porém, o aumento na temperatura não está sujeito unicamente à área de contato entre ombro e junta, mas também depende de quanto do ombro entra em contato com o aço. À medida que o deslocamento da ferramenta aumenta, uma maior porção do ombro entra em atrito com o aço, elevando a temperatura substancialmente. Este aumento torna o metal plastificado mais instável, fazendo com que ele se aglomere na ferramenta e não na junta.

Para verificar estas hipóteses foram comparadas duas juntas soldadas com deslocamentos de - 0,$2 ; 0,0$ e $+0,2 \mathrm{~mm}$, apresentadas na Figura 6. O aporte térmico (AT) durante estas soldagens foi calculado baseado na potência exercida pela máquina $\mathrm{P}\left[\mathrm{J} . \mathrm{s}^{-1}\right], \mathrm{o}$ torque T [N.m], a velocidade de rotação $\omega$ [rpm], e a velocidade de avanço $\cup\left[\mathrm{mm} \cdot \mathrm{min}^{-1}\right]$, segundo as equações seguintes:

$$
P=\frac{T \omega}{60} A T=\frac{P}{v}
$$

Os resultados são apresentados no quadro em vermelho na Figura 6. Comparando as Figura 6a-b é evidente como os deslocamentos e as profundidades maiores da ferramenta geraram quantidades de calor maiores, o qual repercute diretamente no aspecto superficial da junta soldada, confirmando as hipóteses discutidas anteriormente. 


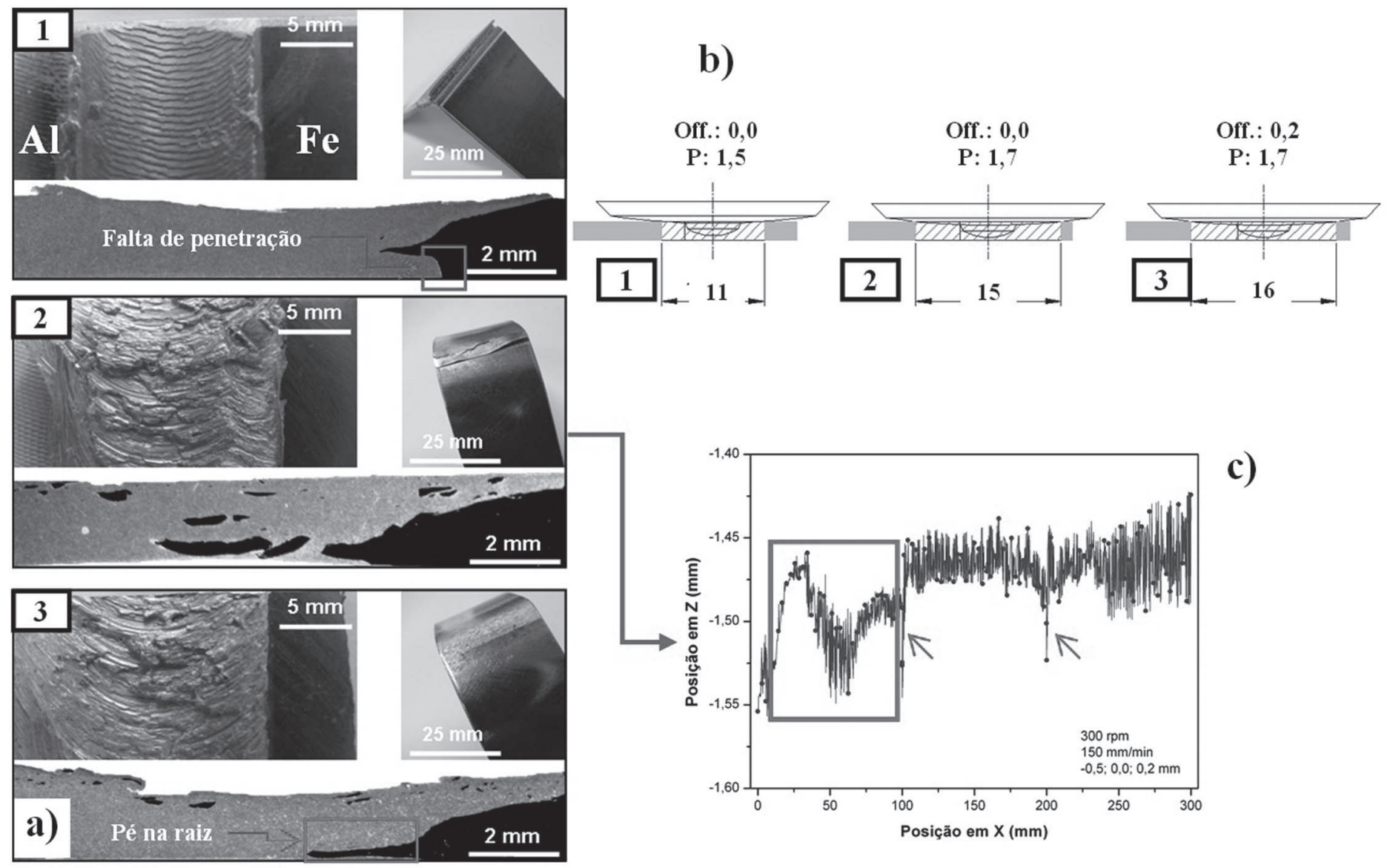

Figura 4. a) Superfícies de soldagem, testes de dobramento e macrografias da seção transversal de juntas soldadas preliminares para as condições da Tabela 2. b) Esquema do efeito da penetração do pino e do deslocamento da ferramenta na área de contato e a penetração atingida durante a soldagem.
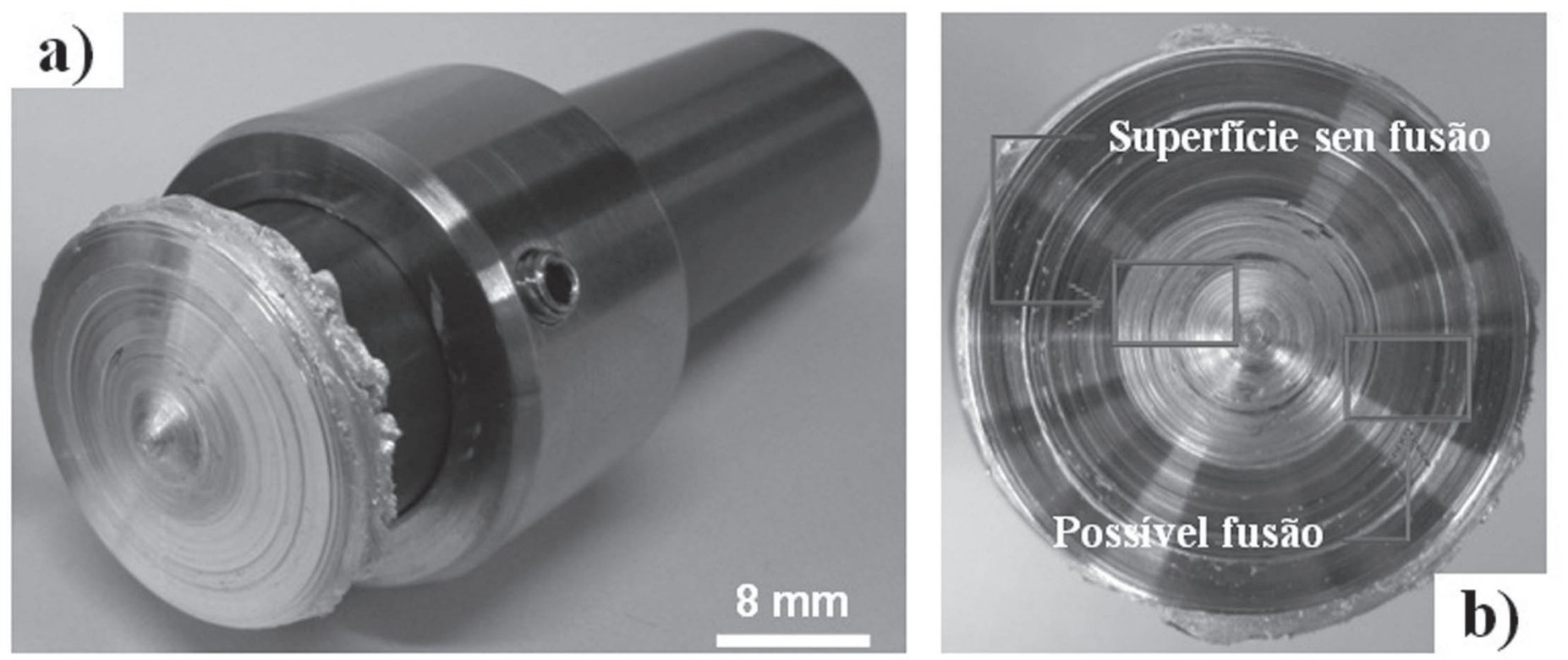

Figura 5. a) Estado final das ferramentas com crosta de alumínio, b) diferencias na superfície indicando possível fusão parcial durante a soldagem. 
Adicional ao efeito no aspecto superficial das juntas soldadas quando há aderência de alumínio, está a perda de estabilidade no processo de soldagem. As forças se tornam instáveis à medida que o alumínio se adere à ferramenta, o que impede o controle do processo através da força axial. Um exemplo disto é a curva da posição em Z, mostrada na Figura 4c. As quedas bruscas na profundidade da ferramenta correspondem a instantes nos quais o alumínio aderiu à ferramenta. $\mathrm{O}$ controle na área de contato permite a redução do calor gerado, melhor acabamento superficial e maior estabilidade do processo, o que é exemplificado na Figura 6a. O melhor acabamento superficial é obtido quando a largura do cordão de soldagem se encontra entre 11 e $13 \mathrm{~mm}$. A Tabela 3 resume as condições de soldagem e as principais observações tanto na junta soldada quanto durante a soldagem. Os resultados mostram como a profundidade atingida pela soldagem aumenta com o deslocamento, tornando possível a junção de chapas de 2,0 $\mathrm{mm}$ com a ferramenta definida. No entanto, esta última medida deve ser tomada com cuidado, evitando aumento drástico na geração de calor, para o qual é conveniente aumentar o deslocamento com a diminuição da área de contato da ferramenta.

As curvas de força e penetração da Figura 6c-d, obtidas na soldagem de juntas usando os modos de controle de posição e controle de força, permitem estabelecer o efeito destes modos de soldagem na penetração na junta soldada. Convencionalmente o modo de soldagem por controle de posição é realizado inicialmente para definir os parâmetros de soldagem, entre estes a força axial a ser utilizada no modo de soldagem por controle de força, que é o utilizado para a soldagem final. Da Figura 6c-d é claro que no modo de controle de posição a força sob a ferramenta muda para manter a penetração; o contrário acontece no modo de controle de força. Neste último a carga oscila de forma constante ao redor de $3800 \mathrm{~N}$ (valor definido para a soldagem), mas as mudanças na penetração, particularmente no caso de soldagem de ligas de alumínio de chapas finas, resulta definitivo para a formação de falta de preenchimento na raiz. Isto indica que o modo de soldagem por controle de força é mais segura para a obtenção de juntas sem defeitos para chapas finas.
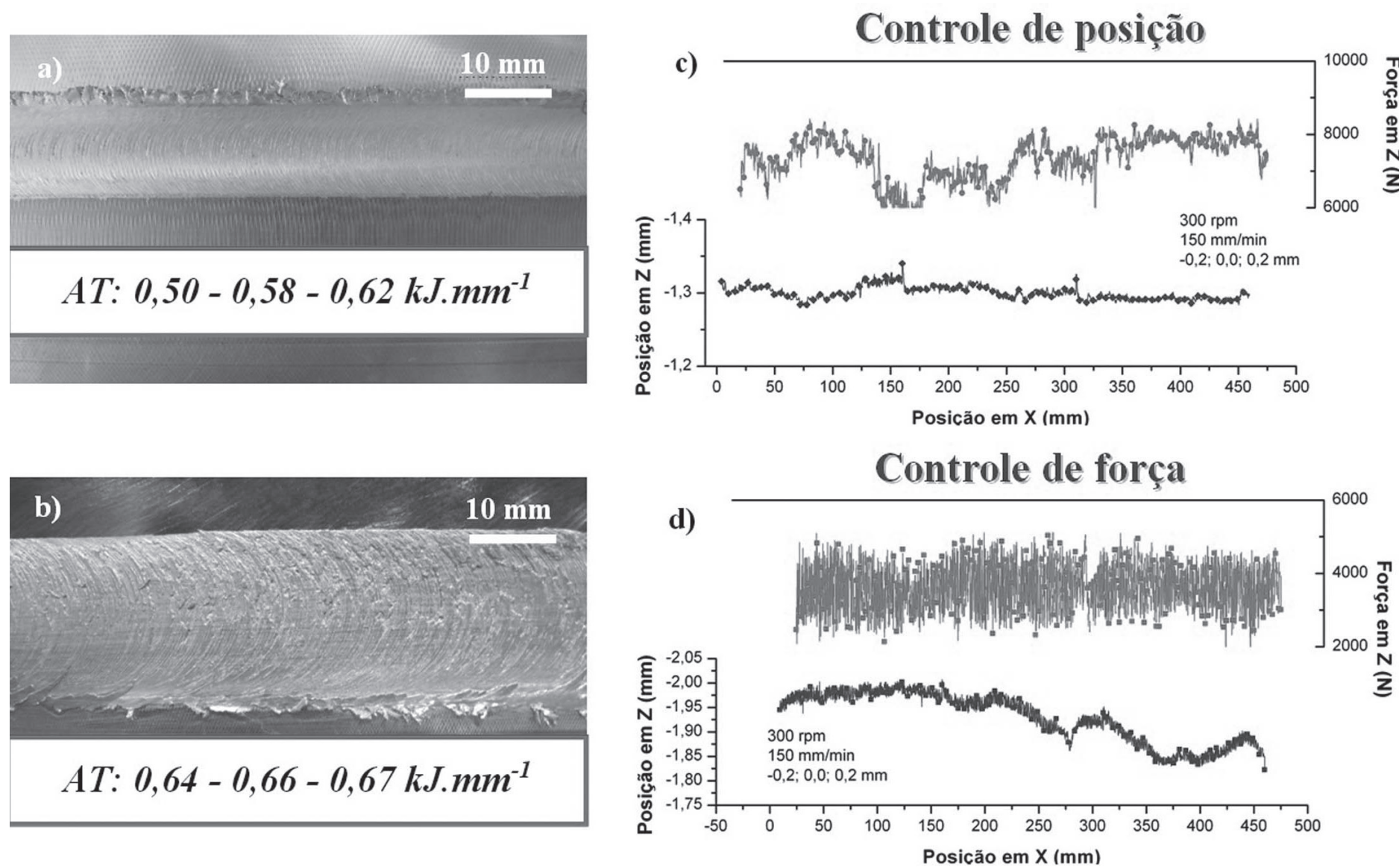

Figura 6. a)-b) Imagens das superfícies de soldagem c)-d) gráficos de força axial e profundidade do pino. No retângulo aparece o AT calculados para deslocamento de $-0,2 ; 0,0 ;+0,2 \mathrm{~mm}$. 
Tabela 3. Parâmetros de soldagem, condições na junta soldada, na ferramenta e no processo durante a SAPNC de juntas dissimilares Al-aço em chapas de 2,0 $\mathrm{mm}$ de espes

\begin{tabular}{|c|c|c|c|c|c|c|c|}
\hline Junta & offset & $\mathbf{P}[\mathbf{m m}]$ & AS & $\mathbf{P A}[\mathbf{m m}]$ & $\mathbf{L}[\mathbf{m m}]$ & Aderência & Processo \\
\hline$(1)$ & 0,00 & 1,5 & Bom & 1,2 & 12 & Não & Estável \\
\hline (2) & 0,00 & 1,7 & Regular & 1,8 & 15 & \multirow{2}{*}{ Sim } & \multirow{2}{*}{ Instável com Vibração da máquina } \\
\hline$(3)$ & 0,20 & 1,7 & Regular & 2,0 & 16 & & \\
\hline \multicolumn{8}{|c|}{$\begin{array}{l}\text { Nota: } \\
\text { P: penetração } \\
\text { AS: acabamento superficial } \\
\text { PA: Penetração atingida } \\
\text { L: Largura da junta }\end{array}$} \\
\hline
\end{tabular}
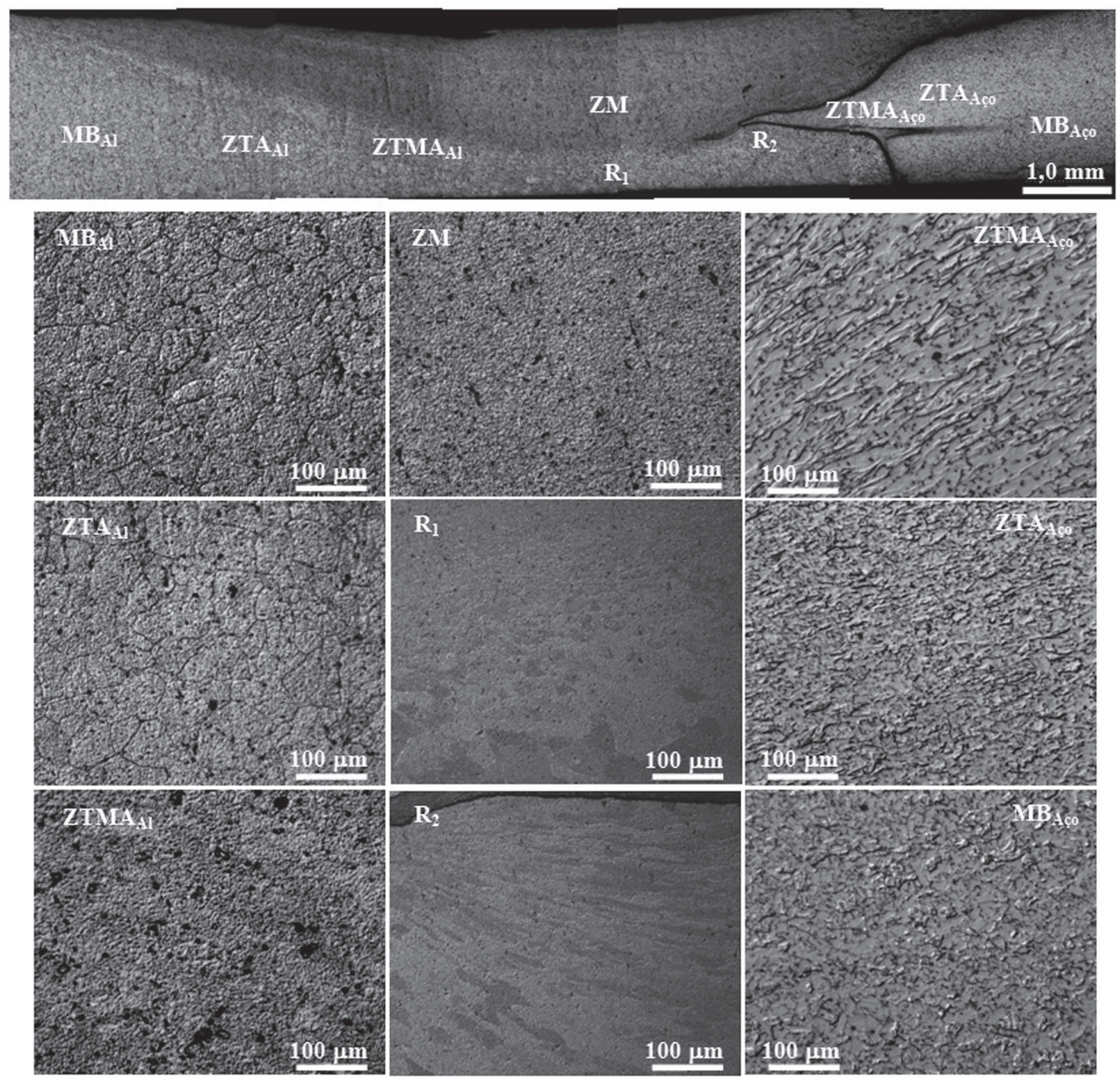

Figura 7. Imagens de MO das diferentes regiões na junta seção transversal de junta soldada a $300 \mathrm{rpm} 150 \mathrm{~mm} \cdot \mathrm{min}^{-1} \mathrm{e} 0,0 \mathrm{~mm}$ de offset (Figura 4a-1). A amostra foi atacada com nital 2\% e HF 1\%. 

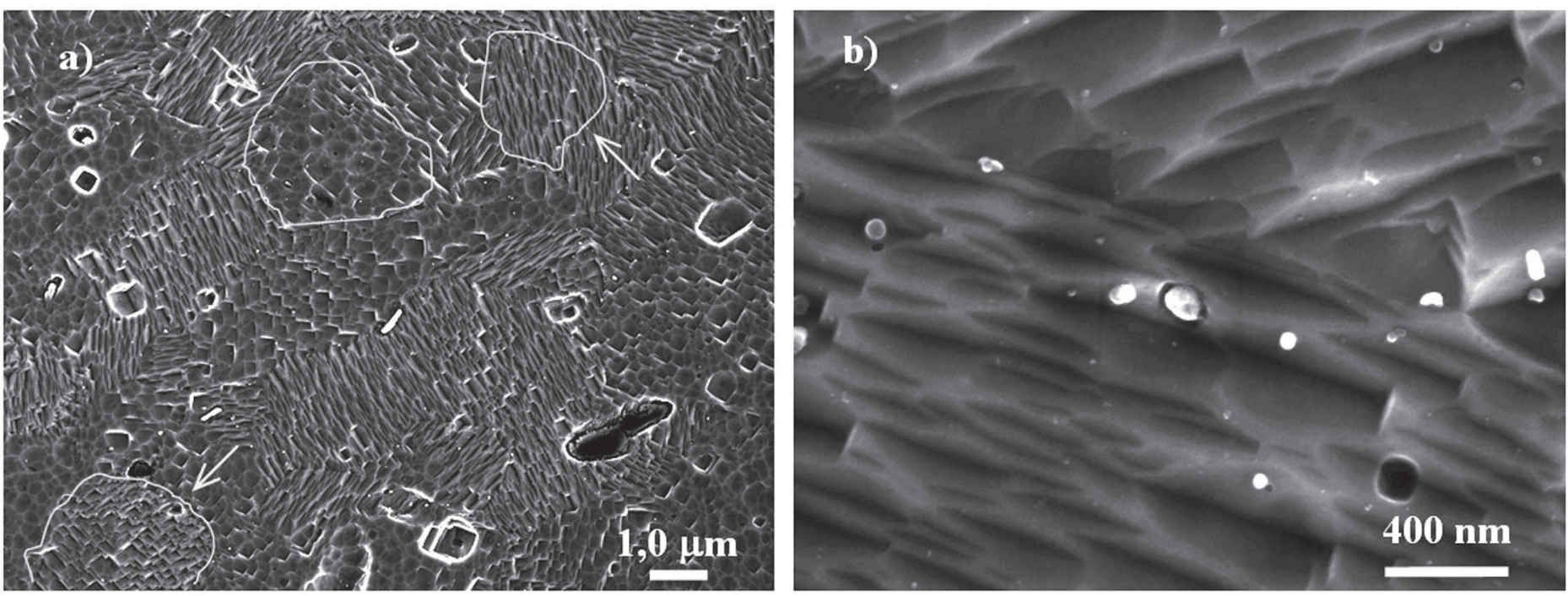

Figura 8. Características microestruturais observadas na ZM. a) Grãos micrométricos entre 0,5 e $2,0 \mu \mathrm{m}$, em amarelo alguns grãos demarcados para melhor identificação; b) precipitados finos de AlFeSi observados principalmente perto do contorno de grão. Imagens obtidas por FEG-MEV no modo SE.

Finalmente as juntas soldadas foram observadas por $\mathrm{MO}$ e MEV para caracterização das diferentes regiões. Em geral as juntas soldada estão formadas por seis regiões designadas como: metal de base do alumínio $\left(\mathrm{MB}_{\mathrm{Al}}\right)$, zona termicamente afetada do $\mathrm{Al}\left(\mathrm{ZTA}_{\mathrm{Al}}\right)$, zona termo-mecanicamente afetada do $\mathrm{Al}\left(\mathrm{ZTMA}_{\mathrm{Al}}\right)$, zona misturada (ZM), zona termo-mecanicamente afetada do aço $\left(\mathrm{ZTMA}_{\mathrm{Aco}}\right)$, zona termicamente afetada do aço $\left(\mathrm{ZTA}_{\mathrm{Aco}}\right)$, metal de base do aço $\left(\mathrm{MB}_{\mathrm{Aço}}\right)$. Estas são apresentadas na Figura 7, a qual corresponde à microestrutura da junta da Figura 4a1. Do lado do alumínio as características microestruturais são similares as observadas em juntas convencionais de alumínio por SAPNC: (1) ZTA caracterizada pelo crescimento de grão $\alpha_{\mathrm{Al}}$, (2) ZTMA sendo esta uma região estreita com grãos $\alpha_{\mathrm{AI}}$ alongados, (3) ZM sem possibilidade de identificação dos grãos por meio de $\mathrm{MO}$, devido a seu tamanho de grão pequeno.

Da Figura 4a-3 um dos elementos de destaque é o pé formado pela entrada do aço no alumínio. A extensão desse pé está diretamente relacionada com o deslocamento da ferramenta e suscita um comportamento diferente na ZTMA na raiz da junta, como mostras as imagens $R_{1}$ e $R_{2}$ da Figura 7 . $R_{1}$ corresponde a uma região de crescimento de grãos e $\mathrm{R}_{2}$, sempre do lado do pé, a uma zona de intensa deformação plástica. A ZM caracteriza-se pela formação de grãos equiaxiais finos, como mostrado na Figura $8 \mathrm{a}$, destacados em amarelo. Também se observou a presença dos precipitados $\mathrm{AlFeSi}$, agora distribuídos aleatoriamente na matriz (Figura 8b-d). Não foi observada nenhuma mudança nestes precipitados, o que indica que mesmo a deformação severa e o calor gerado na ZM resultam insuficientes para a fusão parcial, com a conseqüente formação de liquação constitucional.

Do lado do aço, o ponto mais importante é a formação de uma ZTMA a qual na soldagem de juntas convencionais de aço é eliminada pela transformação polimórfica ferrita-austenitaferrita, induzido pela alta temperatura do processo. Neste caso como a temperatura de soldagem é menor que a tempera de fusão do alumínio, a deformação gerada no aço permanece.

\section{Conclusões}

Foram avaliados os parâmetros de soldagem para a junção por FSW de juntas dissimilares alumínio-aço em chapas de 2,0 $\mathrm{mm}$ de espessura. Os parâmetros de soldagem selecionados foram o deslocamento (offset) e a penetração da ferramenta, sendo avaliados por três critérios: o aspecto da superfície solda, a penetração na junta e o estado da ferramenta. Deste estudo, podem ser emitidas as seguintes conclusões.

- A obtenção de juntas consolidadas - com penetração total - é um balanço entre a geometria da ferramenta (diâmetro do ombro e comprimento do pino) e o deslocamento da mesma (offset), combinados adequadamente para reduzir o calor gerado na junta e evitar o amolecimento excessivo do alumínio.

- Foram obtidas juntas soldadas com acabamento superficial adequado quando a largura do cordão se encontra entre $11 \mathrm{e}$ $13 \mathrm{~mm}$. Foi determinado que o aspecto superficial da junta soldada depende da não aderência do alumínio na ferramenta, o qual está sujeito ao aporte de calor, que por sua vez depende da área de contato entre ombro e junta.

- A aderência do alumínio na ferramenta, além de interferir no aspecto da superfície soldada, reduz drasticamente a espessura e gera defeitos superficiais e volumétricos ao longo da junta. Igualmente, a aderência é responsável pela perda da estabilidade do processo de soldagem, ao gerar quedas drásticas da força axial quando alumínio adere ou se desprende da ferramenta.

- Foi mostrado como para a soldagem de chapas finas o modo de controle por posição resulta mais efetivo para a obtenção de juntas soldadas sem defeitos de raiz. As mudanças de posição no modo de controle por força resultam suficientes para evitar o preenchimento total na junta.

- A SAPNC de juntas dissimilares Al-aço resulta na formação de sete regiões na juntas soldadas: $\left(\mathrm{MB}_{\mathrm{Al}}\right)$, zona termicamente 
afetada do $\mathrm{Al}\left(\mathrm{ZTA}_{\mathrm{Al}}\right)$, zona termo-mecânicamente afetada do Al $\left(\mathrm{ZTMA}_{\mathrm{Al}}\right)$, zona misturada (ZM), zona termomecânicamente afetada do aço $\left(\mathrm{ZTMA}_{\mathrm{Aco}}\right)$, zona termicamente afetada do aço $\left(\mathrm{ZTA}_{\mathrm{Aco}}\right)$, metal de base do aço $\left(\mathrm{MB}_{\mathrm{Aço}}\right)$.

- A formação de ZTMA ${ }_{\mathrm{Aço}}$ neste sistema é de se esperar, devido à alta deformação e baixa temperatura atingidos durante a soldagem, inibindo a transformação polimórfica do aço.

- Os precipitados AlFeSi da liga 6063-T5 se mostram imunes à deformação severa e a temperatura geradas durante a SAPNC.

\section{Agradecimentos}

Os autores agradecem Laboratório Nacional de Luz Síncrotron (LNLS) pelo apoio técnico e financeiro, à Petrobrás pelo apoio financeiro e a FAPESP pela bolsa de estudos.

\section{Referências Bibliografia}

[1] AGUDO, L. et al., Intermetallic FexAly-phases in a steel/Alalloy fusion weld, Journal of Materials Science and Engineering, v. 42, p. 4205-4214, 2007. DOI 10.1007/s10853-006-0644-0.

[2] WATANABE, T. et al., Joining of aluminum alloy to steel by friction stir welding, Journal of Materials Processing Technology, v. 178, p. 342-349, 2006.

[3] CHEN, C.M.; KOVACEVIC., R. Joining of Al 6061 alloy to AISI 1018 steel by combined effects of fusion and solid state welding, International Journal of Machine Tools and Manufacture, v. 44, p. 1205-1214, 2004.

[4] JIANG, W.H.; KOVACEVIC, R., Feasibility study of friction stir welding of 6061-T6 aluminium alloy with AISI 1018 steel, Proceedings of the Institution of Mechanical Engineers, v. 218, p. 1323-1331, 2004.

[5] M. FUKUMOTO, et al. Proceedings of the Fifth International Friction Stir Welding Symposium, France, 2004.

[6] UZUN, H., et al. Friction stir welding of dissimilar Al 6013T4 to X5CrNi18-10 stainless steel, Materials and Design, v. 26, p. 41-46, 2005.

[7] LEE, W. et al., Interfacial reaction in steel-aluminum joints made by friction stir welding, Scripta Materialia, v. 55, p. 355358, 2006.

[8] CHEN, T. J., Process parameters study on FSW joint of dissimilar metals for aluminium-steel, Journal of Materials Science, v. 44, p. 2573-2580, 2009.

[9] CHEN T. P., LIN, W.-B., Optimal FSW process parameters for interface and welded zone toughness of dissimilar aluminumsteel joint. Science and Technology of Welding and Joining v. 15, n. 4, p. 279-285, 2010.

[10] T. YASUI, et al. Proceedings of the Fifth International Friction Stir Welding Symposium, France, 2004.

[11] TANAKA, T., et al., Comprehensive analysis of joint strength for dissimilar friction stir welds of mild steel to aluminum alloys, Scripta Materialia, doi:10.1016/j.scriptamat.2009.06.022.

[12] SOUNDARARAJAN, V., KOVACEVIC, R., Proceedings of the Sixth International Symposium on Friction Stir Welding, Quebec, Canada, 2006.

[13] R. S. COELHO et al, Microstructure and Mechanical Properties of an AA6181-T4 Aluminium Alloy to HC340LA
High Strength Steel Friction Stir OverlapWeld, Advanced Engineering Materials, v.10, n. 10, p. 961-972, 2008.

[14] R. S. COELHO et al, EBSD Technique Visualization of Material Flow in Aluminum to Steel Friction-Stir DissimilarWelding, Advanced Engineering Materials, v. 10, n. 12, p. 1127-1133, 2008.

[15] TOTTEN, G.E.; MACKENZI, D.S. Handbook of Aluminum: Physical Metallurgy and Processes, v. 1, Marcel Dekker, Inc, New York, USA, 2003.

[16] BOURGET, J.-P. et al., Optimization of heat treatment in cold-drawn 6063 aluminium tubes, Journal of Materials Processing Technology, v. 209, p. 5035-5041, 2009.

[17] ZHANG, J. et al., Equilibrium pseudobinary Al-Mg2Si phase diagram, Materials Science and Technology, v.17, p. 494496, 2001.

[18] HEINZ, B. et al., Characterization of a Friction-StirWelded Aluminum Alloy 6013, Metallurgical and Materials Transactions B, v. 33, p. 489-498, 2002.

[19] GAVGALI, M. et al., The effects of artificial aging on wear properties of AA 6063 alloy, Materials Letters, v. 57, p. 37133721, 2003.

[20] AL-MARAHLEH, G. Effect of heat treatment on the distribution and volume Fraction of $\mathrm{Mg} 2 \mathrm{Si}$ in structural aluminum alloy 6063, Metal Science and Heat Treatment, v. 48, n. 5-6, p. 205-209, 2006.

[21] YANG, Y.K. et al., Liquation of Mg alloys in friction stir spot welding, Welding Journal, v. 87, n. 7, p. 167s-177s, 2008. 